\title{
New percolation crossing formulas and second-order modular forms
}

\author{
Nikolaos Diamantis and Peter Kleban
}

\begin{abstract}
We consider the three crossing probability densities for percolation recently found via conformal field theory [23]. We prove that all three of them (i) may be simply expressed in terms of Cardy's [4] and Watts' [24] crossing probabilities, (ii) are (weakly holomorphic) second-order modular forms of weight 0 (and a single particular type) on the congruence group $\Gamma(2)$, and (iii) under some technical assumptions (similar to those used in [19]) are completely determined by their transformation laws.

The only physical input in (iii) is Cardy's crossing formula, which suggests an unknown connection between all crossing-type formulas.
\end{abstract}

\section{Introduction}

Modular behavior appears in percolation theory on a rectangle, as is demonstrated in [19]. There, formulas for the crossing probabilities at the percolation point obtained via conformal field theory (or more exactly, their derivatives with respect to the aspect ratio $r$ ) are shown to have interesting modular properties. Further, a modular characterization of these probabilities is given via several theorems.

In that work, there are two main quantities of interest. The "horizontal" crossing probability $\Pi_{h}(r)$ is the probability of finding at least one cluster that connects the left and right (vertical) edges of the rectangle, with $r$ being the ratio of horizontal to vertical edge lengths. An explicit formula for this quantity was originally calculated by Cardy [4] via conformal field theory, and more recently proven rigorously, for the triangular lattice, by Smirnov [22]. The "horizontal but not vertical" crossing probability $\Pi_{h \bar{v}}(r)$ is the probability of finding at least one cluster that connects the left and right edges of the rectangle while the top and bottom (horizontal) edges are not connected. A formula for this was first obtained with conformal field 
theory by Watts [24] and recently proven rigorously by Dubédat [9] using Schramm-Loewner Evolution methods.

The behavior of the crossing probabilities under $S: z \rightarrow-1 / z$ (with $z=$ $i r$ ) follows from physical symmetries of the problem, while the behavior under $T: z \rightarrow z+1$ arises from the structure of the formulas themselves (explicitly, the appearance of a single "conformal block", see [19] for details), but has no obvious physical origin.

Two surprises arise in [19]. First, there is no reason to expect modular behavior on a rectangle at all since it lacks the appropriate symmetry. (By contrast, on a torus modular behavior of crossing probabilities is both expected and observed ([20]).) Despite this, $\Pi_{h}^{\prime}(r)$ is a modular form (the prime denotes differentiation), and is completely determined by a simple modular argument that assumes its physical symmetry. Secondly, $\Pi_{h \bar{v}}^{\prime}(r)$ is observed to possess unusual modular behavior, which leads to the definition of a new kind of modular object, the nth-order modular form. Such objects were also defined independently, in a different context ([8]) and have since been explored systematically (e.g., $[7,10,13,14,18]$ ).

For percolation, the appearance of a second-order modular form may be traced to the difference in sign of the behavior under $S$ of $\Pi_{h}^{\prime}$ and $\Pi_{h v}^{\prime}$, where $\Pi_{h v}(r)=\Pi_{h}(r)-\Pi_{h \bar{v}}(r)$ is the probability of finding a cluster that crosses both horizontally and vertically.

Recently, three percolation crossing probability densities that can be interpreted in the same setting as $\Pi_{h v}, \Pi_{h \bar{v}}$ (see [19], Section 2) have been calculated from conformal field theory, $\pi_{h}^{b}(\alpha, \beta), \pi_{h}^{b}(\alpha, \beta)$ and $\nu_{h}(\alpha, \beta)([23])$. These quantities (see below for the explicit formulas) are more precisely probability densities for clusters that connect to two specified points on opposite vertical edges of a rectangle, under certain specified conditions. Specifically,

(i) $\pi_{h}^{b}(\alpha, \beta)$ is the probability density of finding at least one cluster that connects two points on the vertical sides of the rectangle, and also connects to the bottom of the rectangle, with no horizontal crossing below it,

(ii) $\pi_{h}^{\bar{b}}(\alpha, \beta)$ is the same except that it is conditioned to not connect to the bottom of the rectangle, and

(iii) $\nu_{h}(\alpha, \beta)$ the probability density of finding at least one cluster that connects two points on the vertical sides of the rectangle conditioned on their being at least one additional horizontal crossing cluster below. 
Here the points on the sides of the rectangle are the images of $\alpha$ and $\beta$, which themselves lie on the real axis. The integrals of these new quantities give $\Pi_{h}, \Pi_{h \bar{v}}$, and $\mathcal{N}_{h}$, where the latter is the expected number of horizontally crossing clusters (see (2.19-2.21) below). (Note that $\mathcal{N}_{h}$ was calculated first via conformal field theory [5,6] and later rigorously [17].)

In this work we examine the modular properties of $\pi_{h}^{b}, \pi_{h}^{\bar{b}}$ and $\nu_{h}$ under the congruence group $\Gamma(2)$. Unexpectedly, we find that all three of them are second-order modular forms. This occurs even though they again are on a rectangle, which does not possess the requisite symmetry under $S$, and they specify crossings between points, rather than intervals. These functions are also very interesting from a purely number-theoretic viewpoint, as they illustrate the occurence of an extension of higher-order forms which involves two group actions and whose study, though formally natural, would seem otherwise unmotivated.

Proposition 2.1 shows that all three crossing probability densities may be written as expressions linear in the ratio $\phi$, which is proportional to $\Pi_{h \bar{v}}^{\prime} / \Pi_{h}^{\prime}$ (see $(2.3),(2.6)$ and (2.13), the prime denotes a derivative), with coefficients that are algebraic functions of the cross-ratio $\lambda$ of the four points defining the rectangle.

Theorem 3.1 proves that all three crossing probability densities are weakly holomorphic second-order modular forms (definitions are given in Section 3), and determines their leading Fourier terms at each cusp. Weak holomorphicity has been studied and led to striking results including the resolution of classical number-theoretical problems on partitions of integers and other results by D. Zagier, R. Borcherds, K. Ono, K. Bringmann ( [1-3,25], see also [11] for further references). However, we did not expect it in this context.

Theorem 4.2 is a uniqueness result for the weakly holomorphic higherorder forms studied in this work. It proves that, under some technical assumptions analogous to those used in [19], all three crossing probability densities are determined by the transformation laws they satisfy. Since the only physical input to the theorem is $\Pi_{h}^{\prime}$, one finds that a single basic framework of assumptions leads to all three probability functions. This points to an unknown connection between the functions $\Pi_{h}, \Pi_{h \bar{v}}$ and $\mathcal{N}_{h}$.

This work is focused on the mathematical aspects of the new crossing probability densities. We plan to explore its physical implications more fully elsewhere. However, since the treatment here may also be of interest to physicists, we have included some comments that are intended to make the presentation more accessible to that audience. 


\section{Crossing probabilities}

Set $T:=\left(\begin{array}{ll}1 & 1 \\ 0 & 1\end{array}\right)$ and $S:=\left(\begin{array}{cc}0 & -1 \\ 1 & 0\end{array}\right)$. We let $\Gamma(2)$ denote the group of matrices of $\mathrm{SL}_{2}(\mathbb{Z})$ congruent to the identity $\bmod (2)$. We use the set of generators of $\Gamma(2)$ consisting of $g_{1}:=T^{2}$ and $g_{2}:=S T^{-2} S^{-1}=\left(\begin{array}{ll}1 & 0 \\ 2 & 1\end{array}\right)$ ([21], p. 63).

Now set $q:=e^{2 \pi i z}$. Let $\eta$ be the Dedekind eta function

$$
\eta(z)=q^{1 / 24} \prod_{n=1}^{\infty}\left(1-q^{n}\right) .
$$

Also, let $\lambda$ denote the classical modular function for $\Gamma(2)$. It is given by

$$
\lambda(z):=16 \frac{\eta(z / 2)^{8} \eta(2 z)^{16}}{\eta(z)^{24}}=1-\frac{\eta(z / 2)^{16} \eta(2 z)^{8}}{\eta(z)^{24}} .
$$

Neither the function $\lambda$ nor its derivative with respect to $z$

$$
\lambda^{\prime}(z)=16 \pi i \frac{\eta(z / 2)^{16} \eta(2 z)^{16}}{\eta(z)^{28}}
$$

have any poles or zeros in $\mathfrak{H}$ (cf. [21], Section 7.2, (7.2.17)).

$\lambda(z)$ appears here because for $z=i r$ it is the cross-ratio of the four points to which the corners of a rectangle of aspect ratio $r$ are mapped.

Since the natural variable in conformal field theory is the cross-ratio $\lambda$, not $r$, we take the variable of the crossing probabilities to be $\lambda$. Indeed, Cardy's formula gives the horizontal crossing probability as

$$
\Pi_{h}(r)=\mathcal{P}_{h}(\lambda(i r))
$$

with $\mathcal{P}_{h}(x):=\frac{2 \pi \sqrt{3}}{\Gamma(1 / 3)^{3}} x^{1 / 3}{ }_{2} F_{1}(1 / 3,2 / 3 ; 4 / 3 ; x)$. Let

$$
C:=\frac{2^{1 / 3} \pi^{2}}{3 \Gamma(1 / 3)^{3}},
$$

following the notation in [19]. Then $\Pi_{h}(r)=\mathcal{P}_{h}(\lambda(i r))$ satisfies

$$
\frac{d}{d r}\left(\mathcal{P}_{h}(\lambda(i r))\right)=-4 \sqrt{3} C \eta(i r)^{4},
$$

as proven in [26]. 
New percolation crossing formulas and second-order modular forms 681

The horizontal but not vertical crossing probability is similarly given by Watt's formula as

$$
\Pi_{h \bar{v}}(r)=\mathcal{P}_{h \bar{v}}(\lambda(i r))
$$

$\mathcal{P}_{h \bar{v}}(\lambda(i r))$ is proven in [19] to satisfy

$$
\frac{d}{d r}\left(\mathcal{P}_{h \bar{v}}(\lambda(i r))\right)=-8 \sqrt{3} f_{2}(i r)
$$

where

$$
\begin{aligned}
f_{2}(z) & =\frac{2 \pi i}{3} \eta(z)^{4} \int_{\infty}^{z} f_{3}(w) d w, \text { with } \\
f_{3}(w) & :=\frac{\eta(w / 2)^{8} \eta(2 w)^{8}}{\eta(w)^{12}} .
\end{aligned}
$$

The Dedekind eta function is a weight $1 / 2$ cusp form on $\mathrm{SL}_{2}(\mathbb{Z})$ with a character. We denote the character of $\eta(z)^{4}$ by $\chi$. As shown in [19], $f_{2}(z)$ is a second-order modular form, of a kind which we specify in the next section.

Now let

$$
g(z):=\eta(z / 2)^{8} \eta(2 z)^{8} / \eta(z)^{16} .
$$

This definition is useful for avoiding ambiguities from fractional powers of $\lambda(1-\lambda)$. Because of $(2.1) g$ satisfies

$$
g^{3}=\frac{1}{16} \lambda(1-\lambda)
$$

Then, combining (2.1) and (2.2) gives, for $z \in \mathfrak{H}$,

$$
\eta(z)^{4}=\frac{1}{16 \pi i} \frac{\lambda^{\prime}(z)}{g(z)^{2}},
$$

as well as

$$
f_{3}(z)=\frac{1}{16 \pi i} \frac{\lambda^{\prime}(z)}{g(z)} .
$$

Now let

$$
\phi(z):=\frac{f_{2}(z)}{\eta(z)^{4}}=\frac{1}{2} C \frac{\left(\mathcal{P}_{h \bar{v}}(\lambda(z))\right)^{\prime}}{\left(\mathcal{P}_{h}(\lambda(z))\right)^{\prime}}
$$

(with prime standing for differentiation in $z$ ). 
By an obvious extension of notation we have $\mathcal{P}_{h v}=\mathcal{P}_{h}-\mathcal{P}_{h \bar{v}}$. Since $\mathcal{P}_{h v}(\lambda(i / r))=\mathcal{P}_{h v}\left(\lambda(i r)\right.$ ) by symmetry, $\Pi_{h v}^{\prime}(i)=0$ (note that $r=1$ is a square). $\Pi_{h}^{\prime}(i)=\Pi_{h \bar{v}}^{\prime}(i)$ then follows immediately. Thus

$$
\phi(i)=\frac{C}{2} .
$$

Using (2.8) to calculate $\phi^{\prime}$ and (2.12), one sees that $\phi$ is a function of $\lambda$ only. Integrating gives

$$
\phi(z)=\frac{1}{2^{8 / 3}} \lambda(z)^{2 / 3}{ }_{2} F_{1}(1 / 3,2 / 3 ; 5 / 3 ; \lambda(z)) .
$$

In a more recent work ( [23]), the three new crossing probability densities mentioned in the Introduction are introduced and computed. For $0<\alpha<1$, and $1<\beta$, they are given by

$$
\begin{aligned}
& \pi_{h}^{b}(\alpha, \beta)=\frac{(\beta+\alpha)_{2} F_{1}(1,4 / 3,5 / 3,1-\alpha / \beta)-2 \beta}{4 \sqrt{3} \pi \beta^{2}(\beta-\alpha)}, \\
& \pi_{h}^{\bar{b}}(\alpha, \beta)=\frac{(\beta+\alpha)_{2} F_{1}(1,4 / 3,5 / 3, \alpha / \beta)+2 \beta}{4 \sqrt{3} \pi \beta^{2}(\beta-\alpha)} \text { and } \\
& \nu_{h}(\alpha, \beta)=\frac{\beta^{2}+2 \alpha \beta-\left(\beta^{2}-\alpha^{2}\right)_{2} F_{1}(1,4 / 3,5 / 3, \alpha / \beta)}{4 \sqrt{3} \pi \beta^{2}(\beta-\alpha)^{2}} .
\end{aligned}
$$

In [23] it is shown that a double integration of each of these quantities gives the crossing probabilities studied in [19] as well as $\mathcal{N}_{h}$. Specifically

$$
\begin{aligned}
\frac{1}{2} \mathcal{P}_{h \bar{v}}(\lambda) & =\int_{0}^{\lambda} \int_{1}^{\infty} \pi_{h}^{\bar{b}}(\alpha, \beta) d \beta d \alpha, \\
\mathcal{P}_{h}(\lambda)-\frac{1}{2} \mathcal{P}_{h \bar{v}}(\lambda) & =\int_{0}^{\lambda} \int_{1}^{\infty} \pi_{h}^{b}(\alpha, \beta) d \beta d \alpha \text { and } \\
-\frac{1}{2} \mathcal{P}_{h \bar{v}}(\lambda)+\frac{\sqrt{3}}{4 \pi} \log \left(\frac{1}{1-\lambda}\right) & =\int_{0}^{\lambda} \int_{1}^{\infty} \nu_{h}(\alpha, \beta) d \beta d \alpha .
\end{aligned}
$$

(The lhs of (2.21) is equal to $\mathcal{N}_{h}(\lambda)-\mathcal{P}_{h}(\lambda)$.)

For clarity, we now introduce the notation

$$
\begin{aligned}
p_{\bar{b}}(z) & :=\pi_{h}^{\bar{b}}(\lambda(z), 1), \\
p_{b}(z) & :=\pi_{h}^{b}(\lambda(z), 1) \quad \text { and } \\
v(z) & :=\nu_{h}(\lambda(z), 1),
\end{aligned}
$$


New percolation crossing formulas and second-order modular forms 683

for the holomorphic functions of $z \in \mathfrak{H}$ obtained from $(2.16),(2.17)$ and (2.18) after we replace $\alpha$ and $\beta$ with $\lambda(z)$ and 1 , respectively.

We next prove

Theorem 2.1. For all $z \in \mathfrak{H}$,

$$
\begin{aligned}
\lambda^{\prime}(z) p_{\bar{b}}(z)= & 4 \sqrt{3} i\left(\frac{\lambda(z) f_{2}(z)}{\lambda^{\prime}(z)}\right)^{\prime} \\
\lambda^{\prime}(z) p_{b}(z)= & 4 \sqrt{3} C i\left(\frac{\lambda(z)}{\lambda^{\prime}(z)} \eta^{4}\right)^{\prime} \\
& -4 \sqrt{3} i\left(\frac{\lambda(z) f_{2}(z)}{\lambda^{\prime}(z)}\right)^{\prime} \text { and } \\
\lambda^{\prime}(z) v(z)= & \frac{\sqrt{3}}{4 \pi}\left(\frac{\lambda(z)}{1-\lambda(z)}\right)^{\prime}-4 \sqrt{3} i\left(\frac{\lambda(z) f_{2}(z)}{\lambda^{\prime}(z)}\right)^{\prime} .
\end{aligned}
$$

Proof. We first deduce from (2.17) that $\pi_{h}^{\bar{b}}(\alpha, \beta)=\beta^{-2} \pi_{h}^{\bar{b}}(\alpha / \beta, 1)$. This and the change of variables $\alpha / \beta \rightarrow \beta$ imply that the rhs of (2.19) equals

$$
\int_{0}^{\lambda} \int_{0}^{\alpha} \pi_{h}^{\bar{b}}(x, 1) d x \frac{d \alpha}{\alpha}
$$

and hence a differentiation in terms of $i r$ together with (2.7) (recalling that the differentiation in (2.7) is in terms of $r$ and not $i r$ ) and re-arranging gives

$$
4 i \sqrt{3} \frac{\lambda(i r) f_{2}(i r)}{\lambda^{\prime}(i r)}=\int_{0}^{\lambda(i r)} \pi_{h}^{\bar{b}}(x, 1) d x
$$

One more differentiation in terms of $i r$ and the definitions (2.22) imply the result for $z=i r$. The analytic continuation of $\pi_{h}^{\bar{b}}(\cdot, 1)$ and the fact that $\lambda, \lambda^{\prime}$ do not have any poles or zeros in $\mathfrak{H}$ imply that it holds for all $z \in \mathfrak{H}$.

The assertions about the other two crossing probability densities are proved in exactly the same way using (2.20) and (2.21).

We next re-write $p_{\bar{b}}, p_{b}$ and $v$ in a way that avoids derivatives of $\lambda$. 
Proposition 2.1. For every $z \in \mathfrak{H}$, we have

$$
\begin{aligned}
p_{\bar{b}}(z) & =\frac{1}{4 \sqrt{3} \pi g(z)^{2}} \frac{1+\lambda(z)}{1-\lambda(z)} \phi(z)+\frac{1}{2 \sqrt{3} \pi} \frac{1}{1-\lambda(z)} \\
p_{b}(z) & =\frac{1}{4 \sqrt{3} \pi g(z)^{2}} \frac{1+\lambda(z)}{1-\lambda(z)}(2 \phi(i)-\phi(z))-\frac{1}{2 \sqrt{3} \pi} \frac{1}{1-\lambda(z)}, \\
v(z) & =-\frac{1}{4 \sqrt{3} \pi g(z)^{2}} \frac{1+\lambda(z)}{1-\lambda(z)} \phi(z)+\frac{1}{4 \sqrt{3} \pi} \frac{1+2 \lambda(z)}{(1-\lambda(z))^{2}} .
\end{aligned}
$$

Proof. By (2.12),

$$
\lambda^{\prime}(z)=16 \pi i g(z)^{2} \eta(z)^{4}
$$

Using (2.10) and (2.26),

$$
3 g^{\prime} g^{2}=\frac{1}{16}(1-2 \lambda) \lambda^{\prime}=\pi i(1-2 \lambda) g^{2} \eta^{4} .
$$

This and (2.26) imply that

$$
\left(\frac{\lambda}{\lambda^{\prime}} \eta^{4}\right)^{\prime}=\left(\frac{\lambda}{16 \pi i g^{2}}\right)^{\prime}=\frac{1}{16 \pi i} \frac{\lambda^{\prime} g-2 g^{\prime} \lambda}{g^{3}}=\frac{\eta^{4}(1+\lambda)}{3(1-\lambda)}
$$

Expanding (2.23) according to this formula, from (2.12) and the definition of $f_{2}$ we deduce

$$
p_{\bar{b}}(z)=\frac{1}{4 \sqrt{3} \pi g(z)^{2}} \frac{1+\lambda(z)}{1-\lambda(z)} \phi(z)+\frac{1}{2 \sqrt{3} \pi} \frac{1}{1-\lambda(z)} .
$$

The proof of the formula for $p_{b}$ is similar. The formula for $v$ may be obtained by comparing (2.17) with (2.18) and making use of the formula for $p_{\bar{b}}$.

Remark 2.1. Using (2.10) to write $g$ in terms of $\lambda$, Proposition 2.1 gives each of $p_{\bar{b}}, p_{b}$ and $v$ as an expression linear in the ratio $\phi$ (see $(2.13)$ ), with the coefficients being rational functions of $\lambda^{1 / 3}$ and $(1-\lambda)^{1 / 3}$. Thus all three probabilities are determined by the same physical quantity $\phi$, which is itself proportional to $\left(\mathcal{P}_{h \bar{v}}(\lambda(z))\right)^{\prime} /\left(\mathcal{P}_{h}(\lambda(z))\right)^{\prime}$. Note also that the constant $C=2 \phi(i)$ in $p_{b}$ is exactly the constant $C$ in (39) of [19]. It follows that $2 \phi(i)-\phi(z)$ and $\phi(z)$ transform into each other under $S$.

Remark 2.2. Proposition 2.1 illustrates why it is natural to work with the group $\Gamma(2)$ rather than the theta group $\Gamma_{\theta}$, as in [19]. The ratio $\phi$ is easily 
seen - use, e.g., (39) of [19] - to transform as a second-order modular form (for the definition see [19] or the next section) under the generators of either $\Gamma(2)$ (e.g., $\left.\left\{g_{1}, g_{2}\right\}\right)$ or $\Gamma_{\theta}$ (e.g., $\left.\left\{g_{1}, S\right\}\right)$. However, $\left.\lambda\right|_{0} S=1-\lambda$; as a result the rational functions of $\lambda$ of Remark 2.1 do not transform simply under $\Gamma_{\theta}$.

\section{Higher-order forms}

Let $\Gamma$ be a congruence subgroup of $\mathrm{SL}_{2}(\mathbb{Z})$ and $k \in 2 \mathbb{Z}$. As usual, for every multiplicative character $\psi$ on $\Gamma$ we define an action of $\Gamma$ on the space of functions $f: \mathfrak{H} \rightarrow \mathbb{C}$ given, for $\gamma \in \Gamma$, by

$$
\left(\left.f\right|_{k, \chi} \gamma\right)(z)=f(\gamma z) j(\gamma, z)^{-k} \overline{\psi(\gamma)}
$$

Here, $j\left(\left(\begin{array}{ll}* & * \\ c & d\end{array}\right)\right):=c z+d$. When $\psi$ is the trivial character $\mathbf{1}$ we write $\left.f\right|_{k} \gamma$.

To describe the condition on the "growth at the cusps", which will be included in the definition of higher-order forms we first note that a cusp is a point $x \in \mathbb{R} \cup\{\infty\}$ such that $\gamma x=x$ for some $\pm 1 \neq \gamma \in \Gamma$ with $|\operatorname{tr}(\gamma)|=2$. Cusps are important because they are the only points on the real line at which modular forms have a regular behavior. Indeed, one of their special features is that if a function is $\Gamma$-invariant under the action $\left.\right|_{k}$, then its composition by an appropriate map sending infinity to a cusp will be periodic and hence it will possess a Fourier expansion. This fact is crucial in establishing the finite dimensionality of the space of modular forms. As a result (as will be seen in the proof of Theorem 4.1) in order to uniquely determine functions, in addition to the transformation laws satisfied, detailed knowledge of the growth at the cusps is essential.

Two cusps $\mathfrak{a}, \mathfrak{b}$ are called equivalent if there is a $\gamma \in \Gamma$ such that $\mathfrak{a}=\gamma \mathfrak{b}$ and inequivalent otherwise.

For each cusp $\mathfrak{a}$ there is a scaling matrix, i.e., a matrix $\sigma_{\mathfrak{a}}$ such that

$$
\sigma_{\mathfrak{a}}(\infty)=\mathfrak{a} \quad \text { and } \quad \sigma_{\mathfrak{a}}^{-1} \Gamma_{\mathfrak{a}} \sigma_{\mathfrak{a}}=\Gamma_{\infty}
$$

where $\Gamma_{\mathfrak{a}}$ (resp. $\left.\Gamma_{\infty}\right)$ is the set of elements of $\Gamma$ fixing $\mathfrak{a}$ (resp. $\left.\infty\right)([12]$, Section 2). We say that a function $f: \mathfrak{H} \rightarrow \mathbb{C}$ is of at most exponential growth (resp. of moderate growth, vanishing) at $\mathfrak{a}$ if there is an $a \in \mathbb{Q}$ (resp. $a \in$ $\left.\mathbb{Q}_{\leq 0}, a \in \mathbb{Q}_{<0}\right)$ such that $\left(\left.f\right|_{k} \sigma_{\mathfrak{a}}\right)(x+i y)=O\left(e^{a y}\right)$ as $y \rightarrow \infty$, uniformly as $x$ ranges over a compact subset of $\mathbb{R}$.

For a set $\left\{\psi_{1}, \ldots, \psi_{n}\right\}$ of characters on $\Gamma$, we define a weakly holomorphic nth-order modular form on $\Gamma$ of weight $k$ and type $\left(\psi_{1}, \ldots, \psi_{n}\right)$ to be a holomorphic function on $\mathfrak{H}$, which is of at most exponential growth at the 
cusps and such that, for every $\gamma_{i} \in \Gamma$,

$$
\left.\left.\left.f\right|_{k, \psi_{1}}\left(\gamma_{1}-1\right)\right|_{k, \psi_{2}}\left(\gamma_{2}-1\right) \ldots\right|_{k, \psi_{n}}\left(\gamma_{n}-1\right)=0
$$

The space of order $n$ weakly holomorphic forms of weight $k$ and type $\left(\psi_{1}, \ldots, \psi_{n}\right)$ on $\Gamma$ is denoted by $\tilde{M}_{k}^{n}\left(\Gamma ; \psi_{1}, \ldots, \psi_{n}\right)$. The subspace of $f \in$ $\tilde{M}_{k}^{n}\left(\Gamma ; \psi_{1}, \ldots, \psi_{n}\right)$ of moderate growth (resp. vanishing) at all cusps is denoted by $M_{k}^{n}\left(\Gamma ; \psi_{1}, \ldots, \psi_{n}\right)\left(\operatorname{resp} . S_{k}^{n}\left(\Gamma ; \psi_{1}, \ldots, \psi_{n}\right)\right)$.

Note that a Fourier expansion at the cusps is not guaranteed. By contrast, in the case of ordinary modular forms, the existence of a Fourier expansion at each cusp is implied by the invariance under the group action but this no longer necessarily holds for orders higher than 1 .

We next prove that the three new crossing probability densities $p_{\bar{b}}, p_{b}$ and $v$ are second-order forms and determine their growth at the cusps.

The group $\Gamma(2)$ has three inequivalent cusps at $\infty, 0$ and -1 . Three corresponding scaling matrices are $I, U:=\left(\begin{array}{cc}0 & -1 \\ 1 & 1\end{array}\right)=S T$ and $U^{2}=\left(\begin{array}{cc}-1 & -1 \\ 1 & 0\end{array}\right)$.

We can then prove

Theorem 3.1. As functions of $z, p_{\bar{b}}(z), p_{b}(z)$ and $v(z)$ are weakly holomorphic second-order modular forms on $\Gamma(2)$ of weight 0 and type $(\mathbf{1}, \chi)$, where $\chi$ is the character associated to $\eta^{4}$. These functions have Fourier expansions at the cusps. The first power of $q$ appearing in the expansion of $p_{\bar{b}}$ at $\infty$ (resp. 0, -1) is 1 (resp. $q^{-5 / 6}, q^{2 / 3}$ ). The corresponding first powers for $p_{b}$ and $v$ are $q^{-1 / 3}, 1, q^{2 / 3}$ and $q^{1 / 2}, q^{-1}, q^{2 / 3}$.

Proof. The function $f_{2}$ is a holomorphic weight 2 second-order form of type $(\mathbf{1}, \chi)$ satisfying

$$
\left.f_{2}\right|_{2}(\gamma-1)=d_{\gamma} \eta^{4} \quad \text { for all } \gamma \in \Gamma_{\theta}
$$

for a constant $d_{\gamma}$ depending solely on $\gamma([19],(38))$. Since $\Gamma(2)$ is a subgroup of $\Gamma_{\theta}$, (3.1) holds for $\Gamma(2)$ too. It is easy to verify that $d_{g_{1}}=0$ and $d_{g_{2}}=$ $C\left(e^{-2 \pi i / 3}-1\right)$, so the rhs of $(3.1)$ is not zero for all $\gamma \in \Gamma(2)$.

An easy computation (see also Theorem 2.2 of [7]) implies that, for $\gamma \in \Gamma(2)$

$$
\left.\left(\frac{\lambda}{\lambda^{\prime}} f_{2}\right)\right|_{0} \gamma=\left.\left.\left(\frac{\lambda}{\lambda^{\prime}}\right)\right|_{-2} \gamma f_{2}\right|_{2} \gamma=\frac{\lambda}{\lambda^{\prime}} f_{2}+\frac{d_{\gamma} \lambda \eta^{4}}{\lambda^{\prime}}
$$

Therefore

$$
\left.\left(\frac{\lambda}{\lambda^{\prime}} f_{2}\right)^{\prime}\right|_{2}(\gamma-1)=\left(\frac{d_{\gamma} \lambda \eta^{4}}{\lambda^{\prime}}\right)^{\prime}
$$


which, by Theorem 2.1, implies

$$
\left.p_{\bar{b}}\right|_{0}(\gamma-1)=d_{\gamma} G
$$

where

$$
G:=\frac{1}{\lambda^{\prime}}\left(\frac{\lambda \eta^{4}}{\lambda^{\prime}}\right)^{\prime} .
$$

Since $\lambda \eta^{4} / \lambda^{\prime}$ transforms as a modular form of weight 0 with character $\chi$ on $\Gamma(2)$, its derivative transforms as a weight 2 form and, therefore, $G$ transforms as a weight 0 form with character $\chi$.

The behavior of $p_{b}$ under the action of $\Gamma(2)$ follows from (2.24), which implies that $p_{b}=-p_{\bar{b}}+4 \sqrt{3} C i G$. Since $\left.G\right|_{0}(\gamma-1)=(\chi(\gamma)-1) G$, we conclude that $\left.p_{b}\right|_{0}(\gamma-1)$ transforms as a weight 0 modular form with character $\chi$.

In the same way, since $(\lambda /(1-\lambda))^{\prime} / \lambda^{\prime}$ transforms as a weight 0 modular form with trivial character, we deduce that $\left.v\right|_{0}(\gamma-1)$ transforms as a weight 0 modular form with character $\chi$.

To complete the proof that $p_{\bar{b}}(z), p_{b}(z)$ and $v(z)$ are second-order weakly holomorphic forms of weight 0 and type $(\mathbf{1}, \chi)$ for $\Gamma(2)$, it remains to show that $p_{\bar{b}}(z)$ and $G$ are of at most exponential growth at the cusps. The argument also proves the last part of the theorem, specifying the first terms of the Fourier expansions of the three probability functions.

We start by determining the first powers of $q$ appearing in the Fourier expansions of $\lambda$ at the three inequivalent cusps $\infty, 0$ and -1 . We shall be making use of (2.11), (2.15), and the transformations of $\lambda:\left.\lambda\right|_{0} S=1-\lambda$ and $\left.\lambda\right|_{0} T=\lambda /(\lambda-1)([21],(7.2 .2))$. Thus

$$
\left.\lambda\right|_{0} U=1 /(1-\lambda) \quad \text { and }\left.\quad \lambda\right|_{0} U^{2}=1-1 / \lambda .
$$

Since the leading term in $\lambda$ at $\infty$ is $16 q^{1 / 2}$, it follows that the leading terms at 0 and -1 are $1+16 q^{1 / 2}$ and $q^{-1 / 2}$, respectively. Therefore $\lambda^{\prime}$ is a weight 2 first-order weakly holomorphic form on $\Gamma(2)$ and the first powers in its Fourier expansions at the cusps $\infty, 0$ and -1 are $q^{1 / 2}, q^{1 / 2}$ and $q^{-1 / 2}$, respectively. The corresponding powers for $\lambda / \lambda^{\prime}$ are then $1, q^{-1 / 2}$ and 1 .

Next we verify that $p_{\bar{b}}(z), p_{b}(z)$ and $v(z)$ have Fourier expansions at $\infty, 0,-1$. We first note that the Fourier expansion of $\lambda$ and (3.4) imply that $\lambda$ maps the cusps $\infty, 0$ and -1 to 0,1 , and $\infty$, respectively. On the other hand, each of the probabilities $\pi_{h}^{\bar{b}}(\lambda(z), 1), \pi_{h}^{b}(\lambda(z), 1)$ and $\nu_{h}(\lambda(z), 1)$ is given (see (2.16), (2.17) and (2.18)) in terms of a hypergeometric function of $\lambda$ or $1-\lambda$ and integral powers of $\lambda$ only. It follows from the classical 
linear transformation formulas for hypergeometric functions that an expansion around any of the cusps will involve integral powers of $\lambda$ or $\lambda^{1 / 3}$ only. Thus any of these expansions is invariant under $T^{6}$, which establishes the periodicity of $g,\left.g\right|_{0} U,\left.g\right|_{0} U^{2}$, for $g=\pi_{h}^{\bar{b}}(\lambda(z), 1), \pi_{h}^{b}(\lambda(z), 1)$ and $\nu_{h}(\lambda(z), 1)$.

The leading term in each of $\pi_{h}^{\bar{b}}(\lambda, 1), \pi_{h}^{b}(\lambda, 1)$ and $\nu_{h}(\lambda, 1)$ and therefore $p_{\bar{b}}(z), p_{b}(z)$ and $v(z)$ at each cusp then follows immediately from (2.16), (2.17) and (2.18) using the Taylor expansion of the hypergeometric function and $\lambda \sim q^{1 / 2}$.

The first terms of the Fourier expansions of $G$ follow in similar (but simpler) way from the Fourier expansions of $\lambda, \lambda^{\prime}$ and the observation that the first power in the expansion of $\eta^{4}$ is $q^{1 / 6}$.

Finally, we must also verify that our functions have at most exponential growth at all equivalent cusps. For $G$, this is automatically implied by its invariance under the action of $\Gamma(2)$. For the three probability functions, it is no longer automatic because they are not $\Gamma(2)$-invariant. Let $\mathfrak{b}=\gamma \mathfrak{a}$ $(\gamma \in \Gamma(2))$ for $\mathfrak{a}=\infty, 0$ or -1 . We have $\sigma_{\mathfrak{b}}=\gamma \sigma_{\mathfrak{a}}, \Gamma_{\sigma_{\mathfrak{b}}}=\gamma \Gamma_{\sigma_{\mathfrak{a}}} \gamma^{-1}$ and that if $\sigma_{\mathfrak{b}}{ }^{\prime}$ is another scaling matrix for $\mathfrak{b}$ then $\sigma_{\mathfrak{b}}=\sigma_{\mathfrak{b}}{ }^{\prime} T^{m}$, with $m \in 2 \mathbb{Z}_{\geq 0}$ ([12], Section 2). Therefore, if $\sigma_{\mathfrak{b}}{ }^{\prime}$ is any scaling matrix of $\mathfrak{b}$, we have (with (3.3))

$$
\left.p_{\bar{b}}\right|_{0} \sigma_{\mathfrak{b}}{ }^{\prime}=\left.p_{\bar{b}}\right|_{0} \sigma_{\mathfrak{b}} T^{m}=\left.p_{\bar{b}}\right|_{0} \gamma \sigma_{\mathfrak{a}} T^{m}=\left.p_{\bar{b}}\right|_{0} \sigma_{\mathfrak{a}} T^{m}+\left.c_{\gamma} G\right|_{0} \sigma_{\mathfrak{a}} T^{m}
$$

From the behaviour of $p_{\bar{b}}$ at $\infty, 0,-1$ we proved above as well as the behavior of $G$ at these cusps, we deduce that $p_{\bar{b}}$ has at most exponential growth at all cusps $\mathfrak{b}$. The same argument applies to $p_{b}$ and $v$.

Table 1: First terms of Fourier expansions

\begin{tabular}{llll}
\hline & $\infty$ & 0 & -1 \\
\hline$\lambda$ & $q^{1 / 2}$ & $1+16 q^{1 / 2}$ & $q^{-1 / 2}$ \\
$\frac{\lambda}{\lambda^{\prime}}$ & 1 & $q^{-1 / 2}$ & 1 \\
$\lambda^{\prime}$ & $q^{1 / 2}$ & $q^{1 / 2}$ & $q^{-1 / 2}$ \\
$\frac{1}{\lambda^{\prime}}$ & $q^{-1 / 2}$ & $q^{-1 / 2}$ & $q^{1 / 2}$ \\
$p_{\bar{b}}(z)$ & 1 & $q^{-5 / 6}$ & $q^{2 / 3}$ \\
$p_{b}(z)$ & $q^{-1 / 3}$ & 1 & $q^{2 / 3}$ \\
$v(z)$ & $q^{1 / 2}$ & $q^{-1}$ & $q^{2 / 3}$ \\
$\left(\frac{\lambda}{\lambda^{\prime}} \eta^{4}\right)^{\prime}$ & $q^{1 / 6}$ & $q^{-1 / 3}$ & $q^{1 / 6}$ \\
$\frac{1}{\lambda^{\prime}}\left(\frac{\lambda}{\lambda^{\prime}} \eta^{4}\right)^{\prime}$ & $q^{-1 / 3}$ & $q^{-5 / 6}$ & $q^{2 / 3}$ \\
\hline
\end{tabular}


New percolation crossing formulas and second-order modular forms 689

For convenience, Table 1 lists the first terms of the Fourier expansions for the functions discussed.

Remark 3.1. Theorem 3.1, in combination with Proposition 2.1, shows that $\phi$ is a weight 0 weakly holomorphic second-order modular form on $\Gamma(2)$ of type $(\mathbf{1}, \bar{\chi})$. This result is already implied in [19], but here it is proved explicitly.

\section{Uniqueness theorems}

We call conformal block of dimension $\alpha \in \mathbb{R}$ a function of $r>0$ expressible in the form

$$
\sum_{n=0}^{\infty} a_{n} e^{-\pi(n+\alpha) r}
$$

with $a_{0} \neq 0$. As noted in [19], if $\Pi(r)$ is a conformal block, then the convergence of (4.1) implies that the function

$$
P(i r):=\Pi(r)
$$

extends to a holomorphic function $P(z)$ with $z \in \mathfrak{H}$ (its power series expansion is exactly as in (4.1), except that $-r$ is replaced by $i z)$. In [19], transformation properties under $S$, which maps the imaginary axis into itself, were important. Here, the corresponding group element is $g_{2}: z \rightarrow z /(1+2 z)$, which does not have this property. Therefore we use a slightly different approach, working exclusively with the analytic continuations of the conformal blocks.

We start with a Lemma which will be needed in the proof of the next theorem.

Lemma 4.1. A function $f \in S_{4}(\Gamma(2), \chi)$ is uniquely determined by the coefficients of $e^{\pi i z / 3}$ in its Fourier expansions at $\infty$ and 0.

Proof of Lemma: For each cusp a, let

$$
E_{\mathfrak{a}}(z):=2 i \lim _{s \rightarrow 1} \frac{d}{d z}\left(\sum_{\gamma \in \Gamma_{\mathfrak{a}} \backslash \Gamma} \operatorname{Im}\left(\sigma_{\mathfrak{a}} \gamma z\right)^{s}\right)
$$


Then

$$
E_{\mathfrak{a}}(z)-E_{\infty}(z)=-1-4 \pi^{2} \sum_{n=1}^{\infty} n\left(Z_{\mathfrak{a}, \infty}(n, 0,1)-Z_{\infty, \infty}(n, 0,1)\right) e^{2 \pi i n z}
$$

where $Z_{\mathfrak{a}, \mathfrak{b}}(m, n, s)$ is the Selberg-Kloosterman zeta function. (Its definition is given in [15], but is rather lengthy. Since we do not use it further, we refrain from quoting it here.) The set $\left\{E_{-1}-E_{\infty}, E_{0}-E_{\infty}\right\}$ spans the direct complement of the space of cusp forms within the space of all modular forms of weight 2 for $\Gamma(2)$ ( [15]). Further, the dimension of $S_{4}(\Gamma(2), \chi)$ is $2([16]$, Section 4.). Therefore a basis is given by $\left(E_{0}-E_{\infty}\right) \eta^{4}$ and $\left(E_{-1}-E_{\infty}\right) \eta^{4}$. As a result, any function $f \in S_{4}(\Gamma(2), \chi)$ has coefficients of $e^{\pi i z / 3}$ in its Fourier expansion at $\infty$ and 0 , which we denote by $a$ and $b$, respectively.

Thus

$$
f=x_{1}\left(E_{0}-E_{\infty}\right) \eta^{4}+x_{2}\left(E_{-1}-E_{\infty}\right) \eta^{4},
$$

for some $x_{1}, x_{2} \in \mathbb{C}$. Further (see [15]), for any cusps $\mathfrak{a}, \mathfrak{b}, \mathfrak{c}$,

$$
j\left(\sigma_{\mathfrak{c}}, z\right)^{-2}\left(E_{\mathfrak{a}}\left(\sigma_{\mathfrak{c}} z\right)-E_{\mathfrak{b}}\left(\sigma_{\mathfrak{c}} z\right)\right)=\delta_{\mathfrak{a} \mathfrak{c}}-\delta_{\mathfrak{b} \mathfrak{c}}+O\left(e^{-2 \pi y}\right),
$$

where $z=x+i y$, which gives

$$
f=-\left(x_{1}+x_{2}\right) e^{\pi i z / 3}+\left.O\left(e^{-7 \pi y / 3}\right) \quad f\right|_{4} U=x_{1} e^{\pi i z / 3}+O\left(e^{-7 \pi y / 3}\right) .
$$

Thus $x_{1}=b$ and $x_{2}=-a-b$.

We are now in a position to prove

Theorem 4.1. Let $F(z)=\sum_{n=0}^{\infty} b_{n} e^{\pi i\left(n+\frac{1}{3}\right) z}$ be the analytic continuation of a conformal block of dimension $1 / 3$, with $b_{0}=\pi i / 3$. Suppose that

(a)

$$
\left.F\right|_{4} g_{2}=\chi\left(g_{2}\right) F,
$$

along some curve in $\mathfrak{H}$,

(b) $F(-1+i / r)$ is bounded as $r \rightarrow \infty$, and

(c)

$$
\lim _{r \rightarrow \infty} e^{\frac{\pi r}{3}} r^{-4} F\left(\frac{i}{r}\right)=-\frac{4}{3} 2^{1 / 3} \pi^{2} .
$$


New percolation crossing formulas and second-order modular forms 691

Then $F(z) \in S_{4}(\Gamma(2), \chi)$ and

$$
F(z)=\frac{\lambda^{\prime}(z)}{\lambda(z)}\left(\frac{\lambda(z) \eta(z)^{4}}{\lambda^{\prime}(z)}\right)^{\prime}
$$

Proof. We first prove that $F$, if it exists, is a weight 4 cusp form for $\Gamma(2)$ with character $\chi$.

Since $F$ is analytic (4.3) holds on all of $\mathfrak{H}$. The Fourier expansion of $F$ and $\chi\left(T^{2}\right)=e^{2 \pi i / 3}$ then imply

$$
\left.F\right|_{4, \chi} T^{2}=F
$$

Therefore, $\left.F\right|_{4, \chi} \gamma=F$, for all $\gamma \in \Gamma(2)$.

We next establish the vanishing at the cusps. First, by its Fourier expansion, $F$ vanishes at $\infty$. To verify that the Fourier expansion of $\left.F\right|_{4} U$ has only positive powers (and thus that $F$ vanishes at 0 ), it suffices to show $\left(\left.F\right|_{4} U\right)(-1+i r) \rightarrow 0$ as $r \rightarrow \infty$. But $\left(\left.F\right|_{4} U\right)(-1+i r)=F(i / r) r^{-4}$, which must vanish as $r \rightarrow \infty$ by (4.4). For the cusp at -1 consider $\left(F \mid{ }_{4} U^{2}\right)(i r)=$ $F(-1+i / r) r^{-4}$. By the assumed boundedness of $F(-1+i / r)$, this vanishes as $r \rightarrow \infty$.

By assumption, the coefficient of $e^{\pi i z / 3}$ in the expansion of $F$ at $\infty$ is

$$
b_{0}=\lim _{z \rightarrow \infty} e^{-\pi i z / 3} F(z)=\frac{\pi i}{3}
$$

The corresponding coefficient at 0 is $\lim _{z \rightarrow \infty} e^{-\pi i z / 3}\left(\left.F\right|_{4} U\right)(z)$. Taking the limit over the line $z=-1+i r$, as $r \rightarrow \infty$, and using (4.4), one has

$$
\lim _{r \rightarrow \infty} F(i / r) r^{-4} e^{\pi r / 3} e^{\pi i / 3}=-\frac{4}{3} 2^{1 / 3} e^{\pi i / 3} \pi^{2}
$$

A computation confirms that these coincide with the corresponding coefficients for $\frac{\lambda^{\prime}}{\lambda}\left(\frac{\lambda}{\lambda^{\prime}} \eta^{4}\right)^{\prime}$. Lemma 4.1 then implies the result.

Remark 4.1. Theorem 4.1 resembles Theorem 1 of [19]. In that case, the assumption of modular transformation properties under $S$ led to a proof that an even conformal block (i.e. one with all $a_{2 n+1}=0$ ) has dimension $1 / 3$ and is in fact equal to Cardy's function $\mathcal{P}_{h}$ (equivalently $\Pi_{h}$, see $(2.3)$ ). Here we assume the block dimension and modular transformation property under the generator $g_{2}$ of $\Gamma(2)$ and find that the function is a simple expression involving $\eta^{4}$, which is proportional to the $z$-derivative of $\mathcal{P}_{h}(\lambda(z))$. The assumption of evenness is not necessary. 
Remark 4.2. Note that if we let $z=i r /(1+2 i r)$ with $r>0$ be the curve in (4.3), the assumption of transformation under $g_{2}$, then the lhs of the equation is in the physical regime, i.e., $z=i r$.

Theorem 4.2. Let $P(z)=\sum_{n=0}^{\infty} a_{n} e^{\pi i(n+1) z}$ be the analytic continuation of a conformal block of dimension 1, and let $F(z)$ be as in Theorem 4.1. Set

$$
P_{1}:=4 \sqrt{3} C i F-P
$$

Further, for a fixed $A \in \mathbb{C}$ set

$$
\tilde{P}(z):=P(z)+A F(z) .
$$

Suppose that (a)

$$
\left.\tilde{P}\right|_{4} g_{2}=\tilde{P}
$$

along a curve in $\mathfrak{H}$ and

(b) $P(-1+i / r)$ is bounded as $r \rightarrow \infty$.

Then

(i) If $A=-C$ and $P(i / r)$ is bounded as $r \rightarrow \infty$,

$$
P(z)=\frac{\left(\lambda^{\prime}(z)\right)^{2}}{\lambda(z)} p_{\bar{b}}(z),
$$

(ii) Under the same assumptions,

$$
P_{1}(z)=\frac{\left(\lambda^{\prime}(z)\right)^{2}}{\lambda(z)} p_{b}(z) .
$$

(iii) If $A=C$ and $P(i / r) r^{-4} \rightarrow-\sqrt{3} \pi / 4$ as $r \rightarrow \infty$,

$$
P(z)=\frac{\left(\lambda^{\prime}(z)\right)^{2}}{\lambda(z)} v(z) .
$$

Proof. We consider the claims in order.

Proof of (i) We proceed exactly as in the proof of Theorem 4.1, except replacing $\chi$ by 1 . Since $\tilde{P}$ (and $P$ ) are analytic, if they exist, (4.5) holds in 
New percolation crossing formulas and second-order modular forms 693

all of $\mathfrak{H}$. Since $g_{2}=S T^{-2} S^{-1},(4.3)$ and $\chi\left(T^{2}\right)=e^{2 \pi i / 3}$ give

$$
\left.P\right|_{4}\left(g_{2}-1\right)=A\left(1-e^{-2 \pi i / 3}\right) F \in S_{4}(\Gamma(2), \chi) .
$$

Further, the Fourier expansion of $P$ implies that $\left.P\right|_{4}\left(T^{2}-1\right)=0$. Hence

$$
\left.P\right|_{4}(\gamma-1) \in S_{4}(\Gamma(2), \chi) .
$$

As in the proof of Theorem 4.1, we have by assumption that $P(i r),\left(P \mid{ }_{4} U\right)$ $(-1+i r)$ and $\left(P \mid{ }_{4} U^{2}\right)(i r) \rightarrow 0$ as $r \rightarrow \infty$, establishing that $P(z)$ vanishes at all three cusps. By (4.7), $\left.P\right|_{4} \gamma(\gamma \in \Gamma(2))$ also vanishes at all three cusps.

Now let

$$
L:=\frac{\left(\lambda^{\prime}\right)^{2}}{\lambda} p_{\bar{b}}
$$

Then

$$
\begin{aligned}
(P-L) \mid g_{1} & =P\left|g_{1}-L\right| g_{1} \\
& =P-L,
\end{aligned}
$$

by the periodicity of both $P$ and $L$. Further

$$
\left.(P-L)\right|_{4}\left(g_{2}-1\right)=A\left(1-e^{-2 \pi i / 3}\right) F-\left.\frac{\left(\lambda^{\prime}\right)^{2}}{\lambda} p_{\bar{b}}\right|_{4}\left(g_{2}-1\right),
$$

by (4.6). By (3.3) and Theorem 4.1,

$$
\left.p_{\bar{b}}\right|_{4}\left(g_{2}-1\right)=d_{g_{2}} G=d_{g_{2}} \frac{\lambda}{\left(\lambda^{\prime}\right)^{2}} F
$$

Therefore, since $\left(\lambda^{\prime}\right)^{2} / \lambda$ is invariant under $g_{2}$,

$$
\begin{aligned}
\left.(P-L)\right|_{4}\left(g_{2}-1\right) & =A\left(1-e^{-2 \pi i / 3}\right) F-d_{g_{2}} F \\
& =0
\end{aligned}
$$

using the formula for $d_{g_{2}}$ after (3.1). Thus $P-L$ is invariant under both generators of $\Gamma(2)$. As noted when recalling the definition of cusps, this $\Gamma(2)$-invariance in terms of the action $\left.\right|_{4}$ also implies that $L$ has Fourier expansions at each cusp of $\Gamma(2)$. Theorem 3.1 (see Table 1) implies that it vanishes at each cusp. Since $P$ vanishes as well, so does $P-L$, and the latter is a standard weight 4 cusp form with trivial character for $\Gamma(2)$. Therefore it vanishes $([16])$, establishing the uniqueness of $P$ (and $\tilde{P}$ ). This completes the proof of $(i)$. 
Proof of (ii): This is immediate from $(i)$, Theorem 4.1, and (2.24).

Proof of (iii): Analogous to the proof of $(i)$, except that $L$ is redefined with $v$ replacing $p_{\bar{b}}$. Here, neither $P$ nor $L$ vanishes at the cusp at 0 , but $P-L$ does, which is sufficient.

Remark 4.3. Theorem 4.2 resembles Theorem 3 of [19]. In that case, the assumption of modular transformation properties under $S$ for two conformal blocks of arbitrary dimension led to a characterization of crossing probabilities that generalize $\Pi_{h}$ and $\Pi_{h \bar{v}}$ (equivalently, $\mathcal{P}_{h}$ and $\mathcal{P}_{h \bar{v}}$, see (2.3) and (2.6)). Here, by assuming the block dimensions, modular transformation properties under the generator $g_{2}$ of $\Gamma(2)$, and cusp properties, we reproduce all three new crossing probability densities.

Remark 4.4. It is interesting that the only input to Theorem 4.2 from the physics of the problem is via $F$. The physical input to $F$ comes from $\eta^{4}$, itself proportional to the $z$-derivative of $\mathcal{P}_{h}(\lambda(z))$. Thus, under the assumptions of the theorem, all three crossing probability densities are determined by $\mathcal{P}_{h}\left(\right.$ or $\left.\Pi_{h}\right)$.

Now $F$ determines the inhomogeneous term in the behavior of the higherorder modular form under $g_{2}$. In [19], which first demonstrated the appearance of higher-order forms in percolation, the appearance of a second-order modular form, more particularly the corresponding inhomogeneous term, is due to the difference in sign of the behavior under $S$ of $\Pi_{h}^{\prime}$ and $\Pi_{h v}^{\prime}$. The former arises from duality, and the latter from an obvious rotational symmetry. Here the symmetry (or other feature) giving rise to higher-order behavior is not clear. Finding a physical interpretation for it may therefore reveal properties of interest.

\section{Acknowledgments}

N.D. thanks Marvin Knopp for useful conversations on certain aspects of the paper. P.K. is grateful to Don Zagier for leading us to Proposition 2.1, for other useful conversations, and for his kind hospitality at the Collège de France, where part of this research was done. He also thanks C. Hongler for an advance copy of [17] and J. J. H. Simmons and R. M. Ziff for useful conversations. Finally, we thank the referee for a careful reading of the paper.

This work was supported in part by the National Science Foundation Grant No. DMR-0536927 (P.K.). 
New percolation crossing formulas and second-order modular forms 695

\section{References}

[1] R. Borcherds, Automorphic forms on $O_{s+2,2}(\mathbb{R})$ and infinite products, Invent. Math. 120(1) (1995), 161-213.

[2] K. Bringmann and K. Ono, The $f(q)$ mock theta function conjecture and partition ranks, Invent. Math. 165 (2006), 243-266.

[3] K. Bringmann and K. Ono, Arithmetic properties of coefficients of halfintegral weight Maass-Poincaré series, Math. Ann. 337(3) (2007), 591612 .

[4] J. L. Cardy, Critical percolation in finite geometries, J. Phys. A 25 (1992) 201-206.

[5] J. Cardy, Conformal invariance and percolation, preprint (2001).

[6] J. Cardy, Linking numbers for self-avoiding loops and percolation: Application to the spin quantum Hall transition, Phys. Rev. Lett. 84 (2000), 3507-3510.

[7] Y. Choie and N. Diamantis, Rankin-Cohen brackets on higher-order modular forms, Proceedings of the Bretton Woods workshop on Multiple Dirichlet series (AMS Proceedings of Symposia in Pure Mathematics).

[8] G. Chinta, N. Diamantis and C. O'Sullivan, Second-order modular forms, Acta Arith. 103(3) (2002), 209-223.

[9] J. Dubédat, Excursion decompositions for SLE and Watts' crossing formula, Probab. Theory Relat. Fields 134 (2006), 453-488 (doi: 10.1007/s00440-005-0446-3).

[10] A. Deitmar, Higher order group cohomology and the Eichler-Shimura map, J. Reine Angew. Math. 629 (2009), 221-235.

[11] W. Duke and P. Jenkins, Integral traces of singular values of weak Mass forms, Algebra Number Theory 2(5) (2008), 573-593.

[12] N. Diamantis, M. Knopp, G. Mason and C. O'Sullivan, L-functions of second-order cusp forms, Ramanujan J. 12(12) (2006), 327-347.

[13] N. Diamantis and D. Sim, The classification of higher-order cusp forms, J. Reine Angew. Math. 622 (2008), 121-153.

[14] N. Diamantis and R. Sreekantan, Iterated integrals and higher order automorphic forms, Comment. Math. Helv. 81(2) (2006), 481-494. 
[15] D. Goldfeld and C. O'Sullivan, Estimating additive character sums for Fuchsian groups, Ramanujan J. 7 (2003), 241-267.

[16] S. Husseini and M. Knopp, Eichler cohomology and automorphic forms, Illinois J. Math. 15 (1971), 565-577.

[17] C. Hongler and S. Smirnov, Critical percolation: the expected number of clusters in a rectangle, Probability Theory and Related Fields (to appear).

[18] Ö. Imamoglu and Y. Martin, A converse theorem for second-order modular forms of level $N$, Acta Arith. 123(4) (2006), 361-376.

[19] P. Kleban and D. Zagier, Crossing probabilities and modular forms, J. Statist. Phys., 113 (2003), 431-454.

[20] H. Pinson, Critical percolation on the torus, J. Stat. Phys. 75 (1994), $1167-1177$.

[21] R. A. Rankin, Modular forms and functions, Cambridge University Press, Cambridge-New York-Melbourne, 1977.

[22] S. Smirnov, Critical percolation in the plane, C. R. Acad. Sci. Paris Sér. I Math. 333(3) (2001), 239-244.

[23] J. J. H. Simmons, P. Kleban and R. M. Ziff, Percolation crossing formulas and conformal field theory, J. Phys. A: Math. Theoret. 40 (2007), F771-F784.

[24] G. Watts, A crossing probability for critical percolation in two dimensions, J. Phys. A: Math. Gen. 29 (1996), L363-L368.

[25] D. Zagier, Traces of singular moduli, Motives, polylogarithms and Hodge theory, Part I (Irvine, CA, 1998), Int. Press Lect. Ser., 3, I, Int. Press, Somerville, MA, 2002, 211-244.

[26] R. M. Ziff, On Cardy's formula for the critical crossing probability in 2D percolation. J. Phys. A 28 (1995), 1249-1255; Proof of crossing formula for $2 D$ percolation (1995), 6479-6480.

School of Mathematical Sciences

UNIVERSiTy OF NOTTINGHAM

NOTTINGHAM

UK

E-mail address: pmznd@maths.nottingham.ac.uk

LASST and Department of Physics And Astronomy,

UNIVERSITY OF MAINE

Orono, MAINE, ME 04473

USA

ReCEIVED May 12, 2009 